\title{
Thalictrum peruvianum (Ranunculaceae), una especie nueva de Lima, Perú
}

\author{
Thalictrum peruvianum (Ranunculaceae), a new species from Lima, Peru
}

Huber Trinidad' ${ }^{1}$ Asunción Cano ${ }^{1,2}$ y Blanca León ${ }^{1}$

1 Laboratorio de Florística, Depar-
tamento de Dicotiledóneas, Museo
de Historia Natural -Universidad
Nacional Mayor de San Marcos.
Av. Arenales 1256 , Lima 11, Perú.
Email Huber Trinidad:
htrinidadpatricio@gmail.com
2 Instituto de Investigación de Cien-
cias Biológicas Antonio Raimondi
(ICBAR), Facultad de Ciencias
Biológicas, UNMSM.
Presentado:
Aceptado:
Publicado online:
$12 / 12 / 201 / 2011$
$08 / 2012$

\begin{abstract}
Resumen
Se describe e ilustra una nueva especie Thalictrum peruvianum $\mathrm{H}$. Trinidad \& A. Cano (Ranunculaceae) del centro de Perú. Está caracterizada por presentar flores solitarias muy pequeñas que nacen opuestas a las hojas y un estigma alado. Estos caracteres diferencian este nuevo taxón del resto de especies sudamericanas. Palabras Claves: Thalictrum peruvianum, Ranunculaceae, nueva especie, Lima, Perú.
\end{abstract}

\section{Abstract}

A new species, Thalictrum peruvianum $\mathrm{H}$. Trinidad \& A. Cano (Ranunculaceae), is described from central Peru. It is characterized for having very small and solitary flowers that born opposite to the leaves, and by winged stigma. These characters distinguish this new taxon from other South American species.

Key Words: Thalictrum peruvianum, Ranunculaceae, new species, Lima, Peru.

\section{Introducción}

$\mathrm{Al}$ realizar un estudio sobre la flora vascular en bosques de Polylepis presentes en la Reserva Paisajística Nor Yauyos Cochas (RPNYC), se observó una pequeña planta que crecía comúnmente en la zona baja del bosque, después de una minuciosa revisión se llegó a la conclusión de que se trataba de una especie nueva. La muestra colectada fue estudiada en el Laboratorio de Florística del departamento de Dicotiledóneas, Museo de Historia Natural de la Universidad Nacional Mayor de San Marcos.

El género Thalictrum posee alrededor de 190 especies en el mundo (Tamura 1995), distribuido principalmente en regiones templadas (Ziman \& Keener 1989). Son pocas las especies que encuentran su hábitat en tierras sudamericanas; Lourteig (1953) citó cinco especies, cuatro Thalictrum decipiens Boivin, T. longistylum DC., T. podocarpum Kunth ex DC. y T. venturii Boivin, representando la sección Camptogastrum de Boivin (1944), y una, T. cincinnatum Boivin, de la sección Cincinneria; las tres primeras han sido registradas para Perú.

Las especies peruanas (Thalictrum decipiens, T. longistylum y T. podocarpum) se distribuyen a ambos lados de la Cordillera de los Andes, principalmente hacia el lado oriental, entre los 1500-3800 m de altitud. Habitan suelos húmedos dentro de matorrales y áreas boscosas, y son poco frecuentes en campos abiertos.

\section{Tratamiento taxonómico}

\section{Thalictrum peruvianum $\mathrm{H}$. Trinidad \& A. Cano, sp. nov.}

TIPO: Perú. Depto. Lima. Prov. Yauyos. Distrito Miraflores. Localidad: Bosque de Yauyinazo a $3 \mathrm{~km}$ de Piños, 12 18'58,95"S y 75050'58,06”'W. Bosque de Polylepis, $3700-3800 \mathrm{~m}$ de altitud, H. Trinidad 221 (Holotipo: USM). Figuras 1 y 2.

Haec species insignis floribus solitaris; sepalis viridis vel purpureis; ovariis 2-5, stigmatibus alatis. Inter species generis Thalictrio ovariis paucis, sed floris solitari, opposita (versus inflorescentia paniculatis), staminum connectivum supra thecas appendiculatum, rubeus differt.
Hierba perenne, postrada escandente, hasta $80 \mathrm{~cm}$ de longitud. Raíces fasciculadas, engrosadas con ramificaciones fibrosas. Tallo cilíndrico hueco de 0,2-1,2 mm de diámetro, verde a purpúreo, estrías poco visibles, pubescencia glandular distribuida de manera dispersa a lo largo del tallo; entrenudos de $2-9 \mathrm{~cm}$. Hojas alternas, 3(4) - pinnadas, imparipinnadas; lámina de $0,8-4,2 \mathrm{~cm}$ de largo por $1,5-6 \mathrm{~cm}$ de ancho, de forma subtriangular, glandular pulverulento en la superficie de la lámina y con escasos pelos glandulares en las nervaduras, verde a purpúreo en los ápices de los foliolos; peciolo de $0,1-2,4$ $\mathrm{cm}$ de longitud esparcidamente glandular pubescente; vaina de $1-2 \times 0,5-1,2 \mathrm{~mm}$, subamplexicaule 2 - auriculada, de base purpura y borde lobulado blanquecino; foliolo asimétrico de $1,5-5 \times 1-5 \mathrm{~mm}, 3$ - lobulado con los ápices obtusos y lóbulo inferior a veces partidos, discoloras, ligeramente pecioluladas y sin estipelas. Flores solitarias opuestas a las hojas, hermafroditas o unisexuales (polígamo monoicas). Pedúnculo de $0,1-1,8$ $\mathrm{cm}$ de longitud. Sépalos 4 de 1,5-3,5 x 1,2-2 mm, $3-6$ nervado, ovado de ápice obtuso, verde a purpureo. Estambres 7 -17, filamentos filiformes que siguen creciendo después de la antesis de $1-5 \mathrm{~mm}$, rojizas; anteras oblongas de $1,4-2 \mathrm{~mm}$, conectivo prolongado por encima de la antera hasta $0,3 \mathrm{~mm}$ de longitud, rojizas. Pistilos en flores perfectas $2-5$ (en las flores femeninas llega a tener hasta 16 pistilos); ovario comprimido lateralmente de $0,6-1,2 \times 0,3-0,8 \mathrm{~mm}$; estilo corto, $0,1-$ $0,2 \mathrm{~mm}$; estigma triangular alado hasta $3,2 \mathrm{~mm}$, papiloso en toda la superficie. Aquenios $1-3$ de $3,5-4,5 \times 2-2,5 \mathrm{~mm}$, cortamente estipitado, estilo persistente; nervaduras prominentes 3 (4) a cada lado, subparalelas.

\section{Material adicional examinado}

Perú. Depto. Lima. Prov. Yauyos. Distrito Miraflores. Localidad: Bosque de Yauyinazo a 3 kilometros de Piños, $12^{\circ} 18^{\prime} 58,95^{\prime}$ 'S y $75^{\circ} 50^{\prime} 58,06^{\prime \prime W}$. Bosque de Polylepis, 3700 $3800 \mathrm{~m}$ de altitud, H. Trinidad 1888 (USM).

\section{Etimología}

Esta nueva especie presenta flores muy pequeñas en comparación a las otras especies del continente, por su distribución conocida decidimos nombrarla Thalictrum peruvianum. 

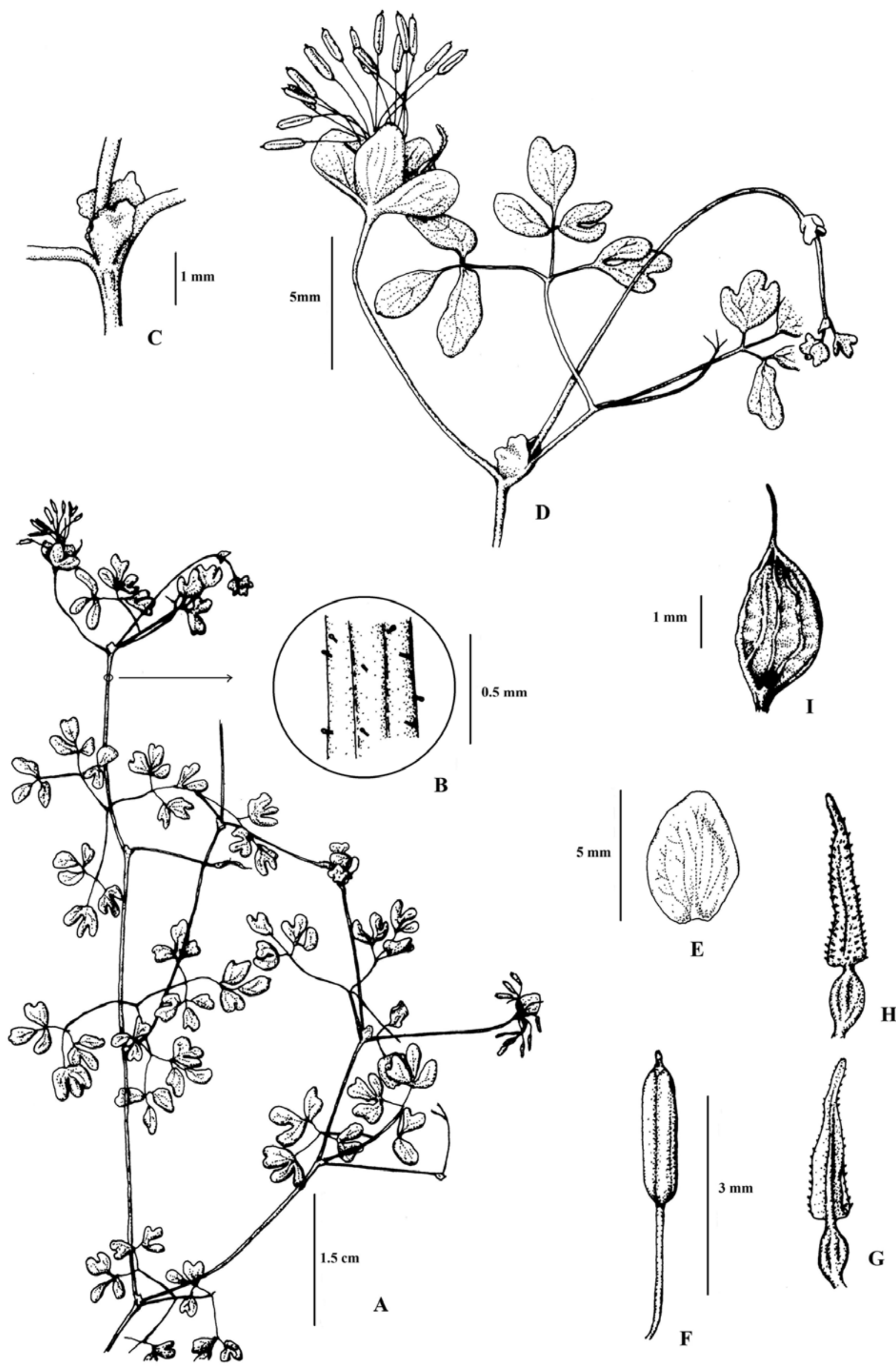

Figura 1. Thalictrum peruvianum $\mathrm{H}$. Trinidad \& A. Cano; a) Habito, b) Segmento de tallo en la que se observa la pubescencia, c) Vaina auriculada sub-amplexicaule, d) Flor perfecta que nace opuesta a la hoja, e) Sépalo, f) Estambre, g) Pistilo con vista dorsal del estigma, h) Pistilo con vista frontal del estigma, e i) Fruto. 


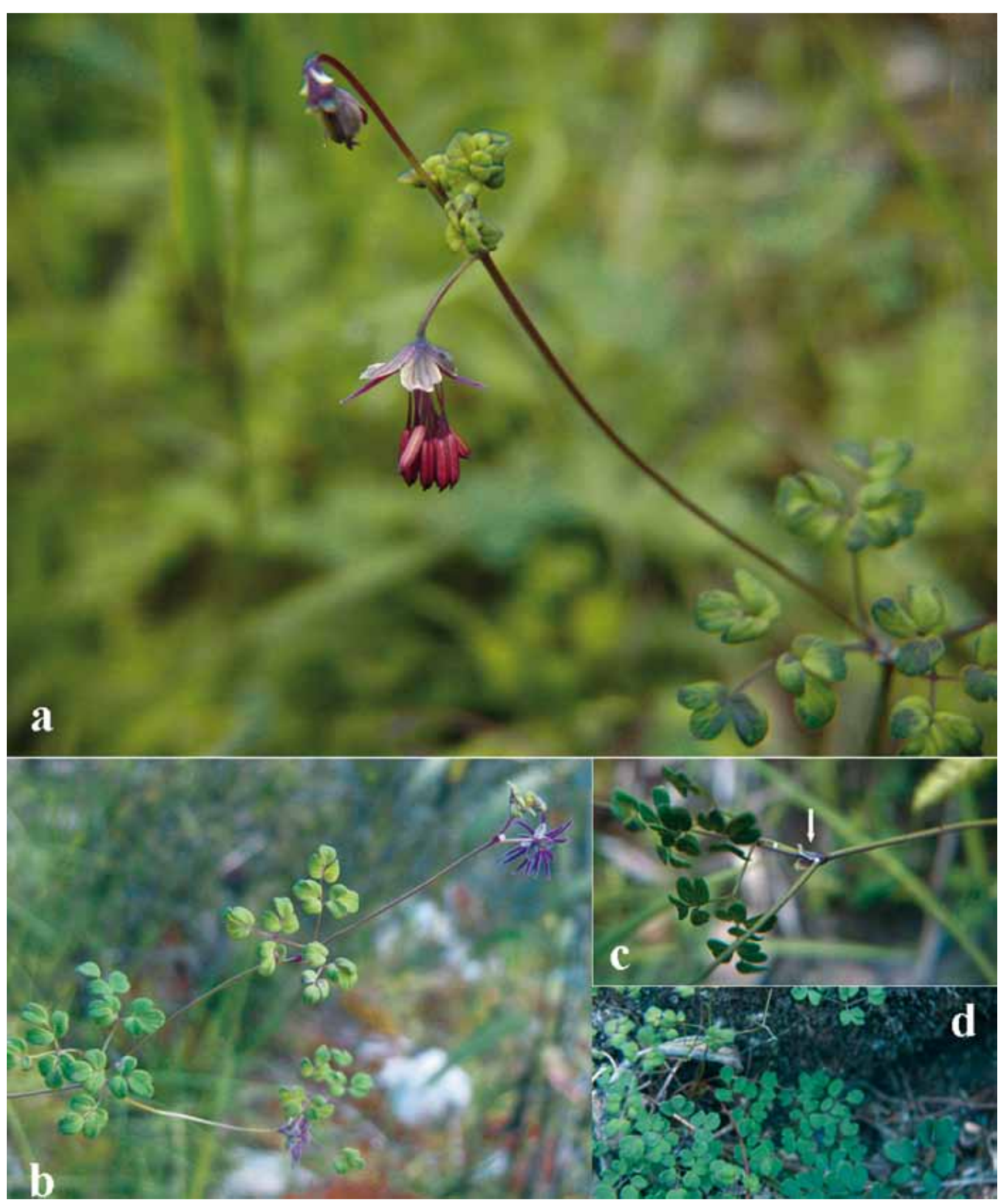

Figura 2. Thalictrum peruvianum $\mathrm{H}$. Trinidad \& A. Cano; a) Rama con flor perfecta, b) Rama con flor femenina, c) Vaina auriculada subamplexicaule y d) Habito. 


\section{Discusión taxonómica}

El género Thalictrum en Perú se distingue fácilmente de los otros géneros de la familia Ranunculaceae por presentar flores con cuatro sépalos y por sus hojas $2-4$ pinnadas.

En Zuloaga et al. (2008) se considera Thalictrum decipiens como el nombre aceptado para las tres especies citadas para el Perú. Pero nosotros concordamos con Lourteig (1953) al decir que Thalictrum decipiens, T. longistylum y T. podocarpum son morfológicamente distintas y que deben ser consideradas como especies diferentes.

Las especies peruanas incluyendo Thalictrum peruvianum se pueden distinguir con la siguiente clave.

1. Flores solitarias. Estilo corto de 0,1-0,2 mm; estigma triangular alado. T. peruvianum

1'. Flores en inflorescencia racemosa terminal. Estilo hasta $10 \mathrm{~mm}$; estigma no triangular alado.

2. Envés de los foliolos con pubescencia glandular.

T. longistylum

2'. Envés de los foliolos glabrescentes.

3. Fruto sésil o sub-sésil con nerviación sub-paralela.

T. decipiens

3'. Fruto estipitado con nerviación reticulada.

T. podocarpum

Thalictrum peruvianum no se encuentra relacionada morfológicamente con ninguna de las especies de Sudamérica; los caracteres florales y foliares que presenta son bastante diferentes. Thalictrum peruvianum es una planta pequeña y crece postrada bajo los arbustos o algunas veces apoyada sobre estos; a diferencia de las otras especies, que crecen erguidas y a veces llegan a ser bastante grandes (2,5 m de altura). El carácter nuevo para el género es que las flores solitarias nacen opuestas a las hojas, hasta ahora se consideraba que todas las especies de este género tenían inflorescencias de disposición terminal o axilar (Macbride 1936; Lourteig 1956; Tamura 1992; Park \& Festerling 1997). Ninguna especie en Sudamérica presenta flores solitarias, aunque como en el caso de T. venturii las inflorescencias pueden llevar pocas flores (Lourteig 1956), pero en esta las flores tienen carpelos estipitados (de 1,5 mm), a diferencia de la especie aquí descrita cuyo estípite es muy corto $(<1 \mathrm{~mm})$. Otro carácter importante y que lo diferencia de todas las especies americanas es la presencia de un estigma triangular alado lleno de papilas. Posiblemente esta sea una adaptación para facilitar la retención de polen debido al pequeño tamaño de sus flores. Este carácter del estigma también ha sido observado en especies asiáticas (Fu \& Zhu 2001), pero estas especies muestran caracteres muy diferentes a $T$. peruvianum.

\section{Distribución, ecología y fenología}

Thalictrum peruvianum ha sido colectada únicamente en un bosque de Polylepis de la RPNYC, el cual es conocido por la población local como bosque de Yauyinazo. Este bosque se encuentra ubicado a lado occidental de la cordillera de los Andes, al sureste de Lima en la provincia de Yauyos; restringida a una quebrada rocosa muy accidentada por encima de los 3700 m de altitud.
Esta especie endémica del Perú se encuentra distribuida en la parte baja del bosque hasta los $3800 \mathrm{~m}$ de altitud; es frecuente ubicarla creciendo postrada en el suelo y algunas veces apoyada sobre los arbustos presentes en la zona.

Florece y fructifica entre los meses de enero y marzo.

\section{Estado de conservación}

Todos los individuos observados han sido hallados en la localidad original, en un bosque de Polylepis. No se ha observado ningún individuo fuera de este bosque, ni en áreas cercanas dentro de la reserva (incluyendo otros bosques evaluados). Se estima que el área total donde se distribuye esta especie no sobrepasa una hectárea de extensión. Se ha observado que existe actividad minera muy cercana al área en la cual se distribuye esta especie. Por lo cual, y de acuerdo a los criterios de la UICN (UICN 2001), se sugiere que sea considerada como una Especie en Peligro Crítico (CR), y se tome las medidas necesarias para poder conservarla.

\section{Agradecimientos}

Agradecemos al Jefe y los guardaparuques de la Reserva Paisajística Nor Yauyos Cochas por permitirnos realizar el trabajo de investigación durante el cual fue posible descubrir esta nueva especie. También nuestra gratitud a Delsy Trujillo por realizar la ilustración que se incluye en esta publicación y a José Roque por la revisión del manuscrito.

\section{Literatura citada}

Boivin, B. 1944. American Thalictra and their Old World allies. Rhodora 46: 391-445.

Fu, D. Z. \& G. Zhu. 2001. Thalictrum. Flora of China, 6: 282-302.

MacBride J. F. 1937. Ranunculaceae, Flora of Peru. Field Museum of Natural History; Botanical Series. 13: 639-661.

Lourteig, A. 1956. Ranunculáceas de Sudamérica Tropical, V. Thalictrum. Mem. Soc. Cienc.Nat. La Salle 16: 186-198.

Park, M. M. \& D. Festerling. 1997. Thalictrum. Flora North America 3: 258-271.

Tamura, M. N. 1993. Ranunculaceae. In: K. Kubitzki, J. G. Rowher, and V. Bittrich, eds. The families and genera of vascular plants. Pp. 563-583. Springer, Berlin.

Tamura, M. N. 1995. Thalictrum. In: P. Hiepko, ed.) Nat. Pflanzenfam. 17a(IV): 474-494. UICN. 2001. Categorías y Criterios de la Lista Roja de la UICN. Versión 3.1. Preparado por la Comisión de Supervivencia de Especies de la UICN. UICN, Gland, Suiza y Cambridge, Reino Unido.

Ziman, S. N. and C. S. Keener. 1989. A geographical analysis of the family Ranunculaceae. Ann. Missouri Bot. Gard.76: 1012-1049.

Zuloaga, F. O; O. Morrone \& M. J. Belgrano. 2008. Ranunculaceae, en F. O. Zuloaga, O. Morrone \& M. J. Belgrano (eds.). Catálogo de las Plantas Vasculares del Cono Sur. Monographs in Systematic Botany from the Missouri Botanical Garden 107 (3): 2825-2839. 